\title{
The Immediate Effects of Neck and Trunk Stabilization Exercises on Balance and Gait in Chronic Stroke Patients
}

\author{
Yu-Won Choe, PT, MS • Myoung-Kwon Kim, PT, $\mathrm{PhD}^{1 \dagger}$ \\ Department of Rehabilitation Sciences, Graduate School, Daegu University, \\ ${ }^{1}$ Department of Physical Therapy, College of Rehabilitation Sciences, Daegu University
}

Received: September 16, 2020 / Revised: September 28, 2020 / Accepted: October 16, 2020

(C) 2020 J Korean Soc Phys Med

\begin{abstract}
| Abstract |
PURPOSE: The purpose of this study was to identify the effects of neck stabilization exercise combined with trunk stabilization exercise on balance and gait function in patients with chronic stroke.
\end{abstract}

METHODS: Twenty-two chronic stroke patients were included in this study. The experimental group subjects ( $\mathrm{n}=$ 11) performed neck stabilization (15 min) and trunk stabilization (15 min) exercises, while the control group subjects $(n=11)$ performed trunk stabilization exercise only for $30 \mathrm{~min}$. Before and after the intervention, the subjects underwent static balance and gait testing.

RESULTS: The 95\% confidence ellipse area, center of pressure (COP) path length, and $\mathrm{COP}$ average velocity were significantly lower in both groups after the intervention compared to before intervention $(\mathrm{p}<.05)$. The average stance force on the affected side increased significantly in both

†Corresponding Author : Myoung-Kwon Kim

skybird-98@hanmail.net, https://orcid.org/0000-0002-7251-6108 This is an Open Access article distributed under the terms of the Creative Commons Attribution Non-Commercial License (http://creativecommons.org/licenses/by-nc/3.0) which permits unrestricted non-commercial use, distribution, and reproduction in any medium, provided the original work is properly cited. groups after the intervention $(\mathrm{p}<.05)$. The changes in the static balance variables were larger in the experimental group than in the control group. The cadence, gait velocity, and single leg support increased significantly in both groups after intervention $(\mathrm{p}<.05)$. The changes in the gait variables were larger in the experimental group than in the control group.

CONCLUSION: Trunk stabilization is a beneficial intervention, but the combination of neck stabilization with trunk stabilization is a more effective method to increase the gait and static balance in chronic stroke patients.

Key Words: Chronic stroke, Balance, Gait, Neck stabilization

\section{Introduction}

Stroke generally leads to the patient having an asymmetrical posture, difficulties of shifting weight to the paralyzed side, and muscle weakening [1,2]. These factors result in low balance ability, abnormal gait, and difficulties in performing various functional activities in stroke patients [3]. This is usually associated with neurological damage to the sensory and motor processes of the postural control system, leading to a decline in the awareness of physical balance, which is required to maintain a precise orientation of the trunk and neck [1,2].

Trunk muscle exercises have been used to improve 
postural control. Trunk control is the ability of the trunk muscles to maintain the body in an upright position, adjust for a shift in weight distribution, and perform selective movements of the trunk to maintain the center of mass within the support base during static and dynamic postural adjustments [4]. The trunk muscles have important roles in various functional activities, such as balance and gait in stroke patients [4,5], and trunk control has been identified as a significant early predictor of a functional outcome following a stroke [6-8]. Core stability training helps activate the deep trunk muscles through selective pelvis movement and abdominal contractions [9]. Trunk stability training through activation of trunk deep muscles improves trunk function, standing balance, and mobility [9]. Several studies have examined the effects of core stability training in stroke rehabilitation, and the authors have provided evidence that trunk control and core stability training can improve balance, mobility, and daily life activities [9-14].

In addition to trunk control, neck control is vital for postural stability and balance [15]. With its high density of proprioceptors, such as muscle spindles and the Golgi tendon organ, the cervical region has an essential role in an individual's ability to recognize their spatial orientation and body position and maintain equilibrium in the changing environments encountered during daily activities [15-17]. In addition, neck muscles exchange relevant information through various nervous system networks, such as the vestibulospinal, vestibulocollic, and the vestibuloocular reflexes, which are involved in controlling the arrangement of the head and trunk [1,18]. Moreover, neck muscles help to stably support the head when holding a gaze $[1,18,19]$ and help maintain whole-body activity $[1,18,20]$. Neck stabilization exercises are reported to activate deep neck muscles [15]. During neck stabilization exercise, select muscles of the neck are contracted, and proprioceptive information from cervical region organs is used [15]. Neck stabilization exercises are reported to improve postural control and balance function $[15,19]$.
Although neck and trunk stability are essential for postural control, balance, and gait $[1,4,15]$, there are no reports on the combined effects of neck and trunk stabilization on balance and gait in chronic stroke patients. To the best of the authors' knowledge, this study is the first to report the combined effects of neck stabilization and trunk stabilization exercises on balance and gait in chronic stroke patients.

\section{Methods}

\section{Participants}

Twenty-two chronic stroke patients who agreed to participate in this experiment were included in this study. Before starting the study, all subjects were informed of its content, and all signed an informed consent form. This study complied with the ethical standards of the World Medical Association Declaration of Helsinki and was approved by the Ethical Committee of Daegu University (1040621-201909-HR-027-02). The subjects of this study were required to meet the following inclusion criteria: 1) first onset of stroke and onset duration of more than six months [15,21]; 2) no cognitive impairment ( $>24$ points on the Mini-mental State Examination-Korean version $[15,21]$; 3) the ability to understand and follow the therapist's directions [21]; 4) ability to stand and walk 10 meters independently and without supervision [15]; 5) an absence of other neurological and orthopedic disorders $[15,21]$.

\section{Experimental procedures}

This study employed a pre- and post-test design. Each subject was assigned randomly to either the experimental group $(\mathrm{n}=11)$ or the control group $(\mathrm{n}=11)$. For randomization, sealed envelopes were prepared in advance and marked inside with an $\mathrm{A}$ or $\mathrm{B}$, indicating the experimental group or the control group, respectively. All tests and interventions were performed in a single day. 


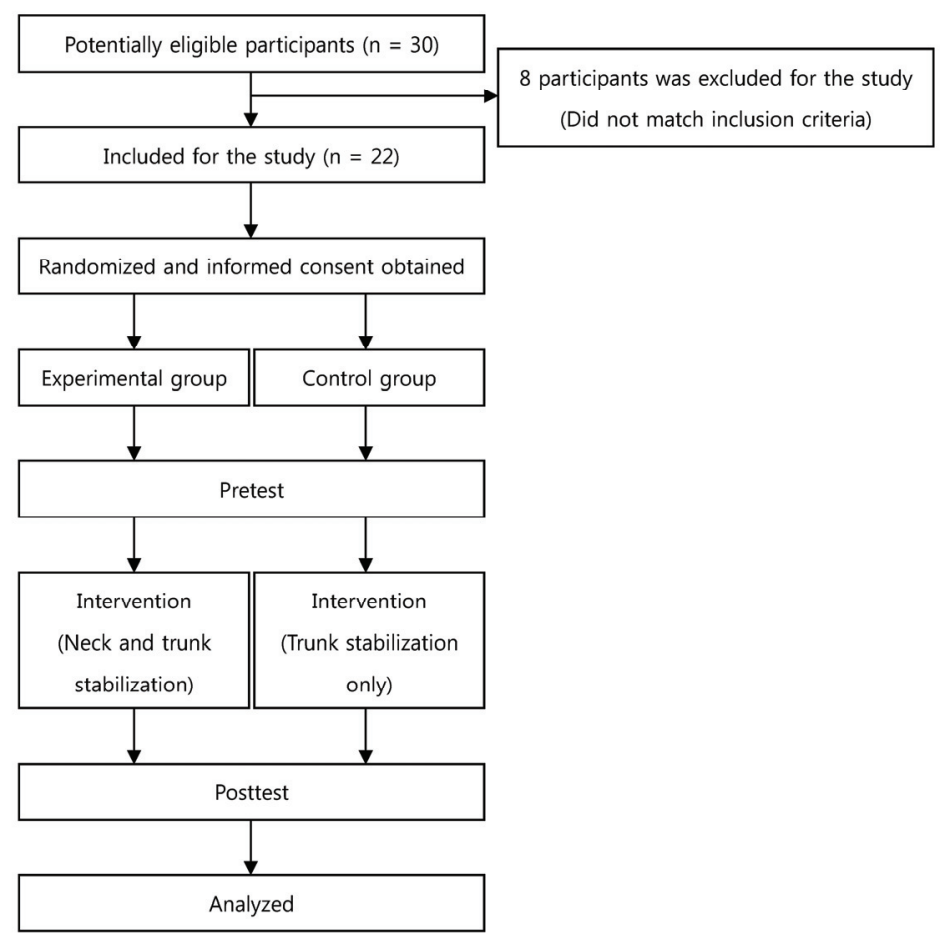

Fig. 1. Study flowchart.

The intervention was provided from $2 \mathrm{pm}$ to $5 \mathrm{pm}$ local time. Before intervention, the subject participated in a pre-test. The subject received the intervention after completing the pre-test, and following the intervention, the subject immediately underwent a post-test. Fig. 1 presents a flowchart of the study procedures. Subjects in the experimental group performed the neck stabilization exercise $(15 \mathrm{~min})$ followed by the trunk stabilization exercise (15 min), whereas the control group subjects performed trunk stabilization exercise only (30 min).

\section{1) Trunk stabilization exercise}

Haruyama et al. [9] developed a step-by-step "core stability training" exercise program that uses select pelvic exercises that were customized for stroke rehabilitation. Their core stability training program consisted of an abdominal drawing-in maneuver (ADIM) to selectively contract the transversus abdominis $(\operatorname{Tr} \mathrm{A})$ muscle, other selective movements of the pelvis, and pelvic movements combined with ADIM. In this program, the level of exercise increases in stages according to a set protocol. For ADIM, the subject draws the lower part of the abdomen up and in toward the spine, without moving the trunk or pelvis and while continuing to breathe normally. The ADIM is performed in a crook lying position and then in the sitting position. Pelvic control exercises include the following three planes of movement: anterior-posterior tilt, lateral lift, and transverse rotation. The selective movements of the pelvis were conducted in the sitting position, and compensatory movements were inhibited. The various motions were performed repeatedly to the maximum range and were voluntarily performed under a low load level. During the pelvic control exercise with ADIM, selective pelvic movements were performed while drawing in the abdomen. If any movement was insufficient, the physical therapist provided additional verbal instructions, manipulative 
induction, or assistance. The effectiveness of the ADIM was judged based on the palpation of a TrA contraction $[9,22]$. All exercises in the sitting position emphasized an upright sitting posture.

\section{2) Neck stabilization exercise}

Craniocervical flexor training was used as a neck stabilization exercise. The craniocervical flexor muscle training process followed the study protocol reported elsewhere $[23,24]$. The neck stabilization exercise targets the deep flexor muscles of the upper cervical region, the longus capitis, and the longus colli muscles, rather than the superficial flexor muscles, the sternocleidomastoid muscle, and the anterior scalene muscle, which flex the neck but not the head $[25,26]$. The exercise is a low-load exercise that specifically trains the deep cervical flexors, rather than all neck flexors, which occurs in the head lift exercise. In this study, the neck stabilization exercise used a sub-occipitally placed air-filled pressure sensor (Stabilizer Pressure Biofeedback Unit, Chattanooga Group Inc., Hixson, TN 37343, USA) to monitor the subtle flattening of the cervical lordosis that occurs with the contraction of the longus colli muscle $[23,27]$. The subject was encouraged by feedback from the pressure sensor to reach five pressure targets sequentially in $2 \mathrm{mmHg}$ increments from a baseline of $10 \mathrm{mmHg}$ to a final level of $20 \mathrm{mmHg}$. The pressure targets used in previous studies ranged from $20 \mathrm{mmHg}$ to $30 \mathrm{mmHg}$, but, in this study, the pressure targets were lowered to a range of $10 \mathrm{mmHg}$ to $20 \mathrm{mmHg}$ because the subjects were chronic stroke patients. The subjects were instructed to gently nod their head as though they were saying 'yes'. The physical therapist identified the target level that the subject could hold steadily for 10 seconds without resorting to retraction, without the dominant use of the superficial neck flexor muscles, and without a quick, jerky craniocervical flexion movement. Contribution from the superficial muscles was monitored by the physical therapist in all stages of the test by using direct observation or palpation. Training commenced at the target level that the subject could achieve with the correct amount of craniocervical flexion and without dominant use or substitution by the superficial muscles (sternocleidomastoid, hyoid, and anterior scalene muscles). The subjects were instructed to perform slow, controlled craniocervical flexion action. They were then trained to sustain progressively increasing ranges of craniocervical flexion based on feedback from the pressure sensor placed behind the neck. For each target level, the contraction duration was increased by 10 seconds, and the subject was trained to perform 10 repetitions. Once this stage was achieved, the exercise progressed to training at the next target level. The exercises were performed to avoid provoking neck pain.

\section{Measurement items}

\section{1) Static balance}

A force platform (FDM SX, Zebris, Germany) was used to measure static balance. This force platform was a 55 $\mathrm{cm} \times 40 \mathrm{~cm}$ plate with a $40 \mathrm{~cm} \times 30 \mathrm{~cm}$ sensor surface with 1,920 pressure sensors. The sampling rate for the device was $120 \mathrm{~Hz}$. Previous studies reported a good ICC $(>.90)$ for this system $[28,29]$. The force platform system measures the center of pressure (COP) sway path length and determines the average COP sway velocity. A longer COP sway path length or higher COP sway velocity indicates inferior static standing balance performance [30]. The confidence ellipse area, which represents the standard deviation of the COP vector [29], and the stance average force, which indicates the weight-bearing proportion of each foot to the whole, were also measured. In this test, all subjects stood on the platform barefoot with their hands next to their bodies and staring at the wall in front of them [29,31]. The location of each foot was recorded to ensure that the feet were positioned in the same place during reassessment testing [31]. During testing with their eyes 
Table 1. General Characteristics of the Subjects

\begin{tabular}{ccccc}
\hline & EG (n = 11) & CG (n = 11) & $t$ & $p$ \\
\hline Age (years) & $66.363 \pm 5.937$ & $65.090 \pm 6.665$ & .473 & .641 \\
Time Since Onset (month) & $17.041 \pm 4.891$ & $17.509 \pm 5.794$ & -.204 & .840 \\
Height (cm) & $161.818 \pm 7.222$ & $162.27 \pm 7.524$ & -.145 & .887 \\
Weight (kg) & $62.272 \pm 10.583$ & $63.363 \pm 8.936$ & -.261 & .797 \\
Gender (male / female) & $5 / 6$ & $6 / 5$ & & \\
Paretic Side (right / left) & $5 / 6$ & $5 / 6$ & & \\
Type of Stroke (ischemia / hemorrhage) & $7 / 4$ & $5 / 6$ & & \\
\hline
\end{tabular}

Mean $\pm \mathrm{SD}$, EG: Experimental group, CG: Control group, ${ }^{*} p<.05$

open, they were asked to stare at a $15 \mathrm{~cm}$ diameter dot placed $3 \mathrm{~m}$ ahead [31]. Data were captured for ten seconds, and five successful trials were recorded with a thirty-second rest interval between trials [29].

\section{2) Gait}

The GAITRite (GaitRite, CIR Systems Inc., USA) system can be used to evaluate temporal parameters, such as velocity, cadence, time of swing phase, and stance phase, as well as spatial parameters, such as stride length, step length, single-limb support, and double-limb support [32]. The GAITRite mat exhibits excellent reliability for most temporospatial gait parameters measured in older subjects [33]. To obtain measurements in this study, the subjects were instructed to stand $3 \mathrm{~m}$ away from the electronic carpet and walk across the carpet at a comfortable walking speed, stopping after walking $3 \mathrm{~m}$ past the electronic carpet [32]. The measurements were repeated three times with a three-minute break between measurements to minimize the potential for bias caused by muscle fatigue [32]. The average value of the three measurements was calculated and recorded [32].

\section{Data processing}

SPSS 20.0 software (SPSS Inc., Chicago, IL, USA) was used for all statistical analyses. A Kolmogorov-Smirnov test and an independent t-test were used to determine the type of distribution for all variables and the intergroup differences, respectively. A paired t-test was used to compare variables before and after the intervention in each group. The selected level of significance was .05 .

\section{Results}

Twenty-two subjects participated in the study (experimental group 11, control group 11). The general characteristics were similar between the two groups ( $p>$ .05) (Table 1).

Compared to before intervention test results, the $95 \%$ confidence ellipse area, COP path length, and COP average velocity were significantly lower in the experimental group and control group after the intervention $(\mathrm{p}<.05)$ (Table 2). The average stance force on the affected side increased significantly in both groups after the intervention $(\mathrm{p}<.05)$ (Table 2). The changes in the static balance variables were larger in the experimental group than in the control group (Table 2), but there was no significant difference between the two groups $(\mathrm{p}>.05)$ (Table 2).

The cadence, gait velocity, and single leg support increased significantly in the experimental and control groups after the intervention $(\mathrm{p}<.05)$ (Table 3$)$. The changes in the gait variables were larger in the experimental group than in the control group (Table 3). However, there were no significant differences between the two groups 
Table 2. Comparison of Static Balance Variables

\begin{tabular}{ccccccc}
\hline & \multicolumn{2}{c}{ EG $(\mathrm{n}=11)$} & & \multicolumn{2}{c}{ CG $(\mathrm{n}=11)$} \\
\cline { 2 - 7 } & Pre-test & Post-test & CWG & Pre-test & Post-test \\
\hline 95\% Confidence Ellipse Area $\left(\mathrm{mm}^{2}\right)$ & $1308.454 \pm 774.656$ & $822.909 \pm 627.034^{*}$ & $-485.545 \pm 335.775$ & $1223.090 \pm 716.953$ & $828.454 \pm 529.592^{*}$ & $-394.636 \pm 248.759$ \\
COP Path Length $(\mathrm{mm})$ & $214.909 \pm 101.903$ & $139.090 \pm 66.846^{*}$ & $-75.818 \pm 60.121$ & $192.363 \pm 65.743$ & $129.909 \pm 42.176^{*}$ & $-62.454 \pm 31.462$ \\
COP Average Velocity $(\mathrm{mm} / \mathrm{sec})$ & $21.363 \pm 10.112$ & $13.818 \pm 6.764^{*}$ & $-7.545 \pm 6.006$ & $19.181 \pm 6.675$ & $12.727 \pm 4.360^{*}$ & $-6.454 \pm 3.077$ \\
$\quad$ Stance & $30.818 \pm 6.968$ & $38.727 \pm 5.605^{*}$ & $7.909 \pm 4.989$ & $32.363 \pm 6.874$ & $38.000 \pm 5.744^{*}$ & $5.636 \pm 3.443$ \\
\hline
\end{tabular}

Mean \pm SD, CWG: Changes within groups, EG: Experimental group, CG: Control group, ${ }^{*}$ Significant difference in gains between pre-test and post-test, $p<.05$

Table 3. Comparison of Gait Variables

\begin{tabular}{cccccccc}
\hline & & EG $(\mathrm{n}=11)$ & & & & & \\
& & & & \\
& Pre-test & Post-test & CWG & & Pre-test & Post-test \\
\hline Cadence (steps/min) & $59.845 \pm 17.567$ & $64.836 \pm 17.744^{*}$ & $4.990 \pm 2.739$ & $56.290 \pm 14.014$ & $60.445 \pm 14.085^{*}$ & $4.154 \pm 2.003$ \\
Gait Velocity (cm/sec) & $23.827 \pm 10.099$ & $29.027 \pm 12.984^{*}$ & $5.200 \pm 3.408$ & $22.563 \pm 10.309$ & $27.109 \pm 12.520^{*}$ & $4.545 \pm 3.533$ \\
Single Leg Support on the Affected Side (\%GC) & $20.018 \pm 6.915$ & $23.772 \pm 8.126^{*}$ & $3.754 \pm 2.507$ & $20.236 \pm 6.924$ & $23.845 \pm 8.168^{*}$ & $3.609 \pm 2.273$ \\
\hline
\end{tabular}

Mean \pm SD, CWG: Changes within groups, EG: Experimental group, CG: Control group, *Significant difference in gains between pre-test and post-test, $\mathrm{p}<.05$

$(\mathrm{p}>.05)($ Table 3$)$.

\section{Discussion}

In this study, the results of the standing balance variables (95\% confidence ellipse area, COP path length, COP average velocity, and stance average force on the affected side) indicated that both the combination of neck stabilization with trunk stabilization and trunk stabilization only are effective methods for achieving standing balance in chronic stroke patients. On the other hand, the intervention that included neck stabilization with trunk stabilization together increased the standing balance to a greater extent. In this study, all the static balance parameters are related to postural control, a sense of equilibrium, and balance responses. A previous study applied both neck and trunk stabilization exercises and reported improved postural control, sense of equilibrium, and orientation response [34]. They observed that neck and trunk stabilization exercises improved the static balance ability in cerebral palsy subjects. Moreover, the neck and trunk were complementary, and the authors indicated that neck stabilization exercises and trunk stabilization exercises should be applied together to improve the balance ability [34]. Another study examined the effects of trunk stabilization exercise only and combined neck and trunk stabilization exercises on the balance ability (COP sway length and limit of stability) in the elderly [18]. They reported that both exercises effectively improved static balance ability, but the combined neck and trunk stabilization exercises were more efficient for increasing balance ability in older adults [18]. Our study also found that performing neck stabilization with trunk stabilization together is more helpful for increasing balance function than trunk stabilization only. The reason why the combination of neck stabilization with trunk stabilization is more effective is because patients undergoing combined treatment receive more information from cervical proprioceptors and the vestibular system during the combination treatment. Previous studies reported that information coming from the body and the environment can be delivered simultaneously to the neck and trunk to enable coordination between head and limb movements, including movement of the neck muscles $[1,34,35]$. One function of the neck muscles is to coordinate the position of the body and head against the surrounding environment and provide a stable base of support for the visual and vestibular systems during 
postural control $[1,34,35]$. Peripheral proprioceptive organs in the cervical region, particularly in the upper cervical muscles, are advantageous for controlling and organizing somatosensory information related to balance [15], and trunk stabilization and balance are helpful in stroke patients [9-14]. Furthermore, if neck stabilization exercises, which use proprioceptive information from cervical region organs, are added to trunk exercises, the combination is a more effective intervention to help adapt to a changing environment by controlling postural perturbations [15].

In this study, the variable results (cadence, gait velocity, and single leg support on the affected side) indicate that both neck stabilization with trunk stabilization and trunk stabilization only are effective methods for improving the gait ability in chronic stroke patients. However, the combination intervention (neck and trunk stabilization) is more helpful for increasing the gait ability. Dubey et al. [36] performed pelvic stability training that included selective contraction and co-activation of the lower trunk and proximal part of the lower extremities in stroke patients and showed significant improvements in gait speed associated with the increased movement control of the trunk and lower extremities. Pelvic movements during walking are altered in stroke, and the changes are related to the gait speed $[37,38]$. Therefore, improved pelvic stability not only has a practical effect on balance, but also allows better stance control and limb advancement, which are the major determinants of gait speed and cadence [36,39]. Previous studies reported that trunk stabilization is important for functional activities, such as balance and gait, in stroke patients $[4,5]$. Similarly, many studies have emphasized the role of neck stabilization in postural control and trunk stabilization [15]. The results from the present study are consistent with those in several prior studies. These results indicate that an intervention that combines neck stabilization with trunk stabilization helps increase gait ability. Moreover, gait ability is related to balance ability, requiring postural control [1]. Therefore, increasing neck and trunk stability has a large effect on gait because neck muscles and information from the neck to the trunk are connected with the visual and vestibular systems associated with postural control $[1,34,35]$.

This study had several limitations. First, the sample size was small. Second, this study could not identify the long term or clinical effects of a combination of neck stabilization and trunk stabilization. Given these limitations, additional research will be needed.

\section{Conclusion}

Trunk stabilization exercise is a beneficial intervention in chronic stroke patients, but a combination of neck and trunk stabilization exercises is more effective than trunk only exercises in improving gait and static balance in chronic stroke patients.

\section{References}

[1] Shumway-Cook A, Woollacott MH. Motor control: translating research into clinical practice (3rd ed). Lippincott Williams \& Wilkins. 2007.

[2] Jeon SH, Lee SM, Kim JH. Therapentic effects of reaching with forward bending of trunk on postural stability, dynamic balance, and gait in individuals with chronic hemiparetic stroke. J Phys Ther Sci. 2015;27(8):2447-51.

[3] Kim H, Her JG, Ko J. Effect of horseback riding simulation machine training on trunk balance and gait of chronic stroke patients. J Phys Ther Sci. 2014;26(1):29-32.

[4] Karthikbabu S, John MS, Manikandan N, et al. Role of trunk rehabilitation on trunk control, balance and gait in patients with chronic stroke: a pre-post design. Neuroscience \& Medicine. 2011.

[5] Verheyden G, Vereeck L, Truijen S, et al. Trunk performance after stroke and the relationship with balance, gait and functional ability. Clin Rehabil. 2006;20(5): 451-8. 
[6] Duarte E, Marco E, Muniesa JM, et al. Trunk control test as a functional predictor in stroke patients. J Rehabil Med. 2002;34(6):267-72.

[7] Hsieh CL, Sheu CF, Hsueh IP, et al. Trunk control as an early predictor of comprehensive activities of daily living function in stroke patients. Stroke. 2002;33(11): 2626-30.

[8] Verheyden G, Nieuwboer A, De Wit L, et al. Trunk performance after stroke: an eye catching predictor of functional outcome. J Neurol Neurosurg Psychiatry. 2007;78(7):694-8.

[9] Haruyama K, Kawakami M, Otsuka T. Effect of core stability training on trunk function, standing balance, and mobility in stroke patients: a randomized controlled trial. Neurorehabil Neural Repair. 2017;31(3):240-9.

[10] Cabanas-Valdés R, Bagur-Calafat $\mathrm{C}$, Girabent-Farrés $\mathrm{M}$, et al. The effect of additional core stability exercises on improving dynamic sitting balance and trunk control for subacute stroke patients: a randomized controlled trial. Clin Rehabil. 2016;30(10):1024-33.

[11] Ting-Ting L, Meng-Jie L, Ya-Qian L, et al. Effects of core stability exercise on rehabilitation in stroke patients with hemiplegia: a meta-analysis. TMR Non-Drug Therapy. 2018;1(2):41-52.

[12] Yu SH, Park SD. The effects of core stability strength exercise on muscle activity and trunk impairment scale in stroke patients. J Exerc Rehabil. 2013;9(3):362.

[13] Chung EJ, Kim JH, Lee BH. The effects of core stabilization exercise on dynamic balance and gait function in stroke patients. J Phys Ther Sci. 2013;25(7):803-6.

[14] Lee JY, Park JS, Lee DH, et al. Effect of the trunk muscle stabilization training on balance for chronic stroke patients. Journal of the Korea Academia-Industrial cooperation Society. 2012;13(3):1212-9.

[15] Kim GM, Oh DW. Neck proprioceptive training for balance function in patients with chronic poststroke hemiparesis: a case series. J Phys Ther Sci. 2014;26(10): $1657-9$.
[16] Boyd-Clark LC, Briggs CA, Galea MP. Muscle spindle distribution, morphology, and density in longus colli and multifidus muscles of the cervical spine. Spine. 2002; 27(7):694-701.

[17] Bove M, Courtine G, Schieppati M. Neck muscle vibration and spatial orientation during stepping in place in humans. J Neurophysiol. 2002;88(5):2232-41.

[18] Song GB, Park EC. Effects of neck and trunk stabilization exercise on balance in older adults. J Kor Phys Ther. 2016;28(4):221-6.

[19] Seo HJ, Lee MJ, Oh TY. The effects of neck strengthening exercise on postural control in sitting position during reaching in children with spastic diplegia. J Korean Soc Neur Ther. 2012;16(16):17-24.

[20] Freitas SM, Duarte M, Latash ML. Two kinematic synergies in voluntary whole-body movements during standing. J Neurophysiol. 2006;95(2):636-45.

[21] Lee JH, Choi JD. The effects of upper extremity task training with symmetric abdominal muscle contraction on trunk stability and balance in chronic stroke patients. J Phys Ther Sci. 2017;29(3):495-7.

[22] Hides J, Richardson C, Hodges P. Local segmental control. In: Therapeutic Exercise for Lumbopelvic Stabilization: A Motor Control Approach for the Treatment and Prevention of Low Back Pain (2nd ed). London, England. Churchill Livingstone. 2004.

[23] Falla D, Jull G, Russell T, et al. Effect of neck exercise on sitting posture in patients with chronic neck pain. Phys Ther. 2007;87(4):408-17.

[24] Jull GA, Falla DL, Treleaven JM, et al. A therapeutic exercise approach for cervical disorders (3rd ed). Edinburgh, United Kingdom. Elsevier. 2004.

[25] Falla D, Bilenkij G, Jull G. Patients with chronic neck pain demonstrate altered patterns of muscle activation during performance of a functional upper limb task. Spine. 2004;29:1436-40.

[26] Falla D, Jull G, O'Leary S, et al. Further evaluation of an EMG technique for assessment of the deep cervical 
flexor muscles. J Electromyogr Kinesiol. 2006;16:621-8.

[27] Mayoux-Benhamou MA, Revel M, Vallee C, et al. Longus colli has a postural function on cervical curvature. Surg Radiol Anat. 1994;16(4):367-71.

[28] Nakhaee Z, Rahimi A, Abaee M, et al. The relationship between the height of the medial longitudinal arch (MLA) and the ankle and knee injuries in professional runners. The Foot. 2008;18(2):84-90.

[29] Soltani N, Rahimi A, Naimi SS, et al. Studying the Balance of the Coper and Non-Coper ACL-Deficient Knee Subjects. Asian J Sports Med. 2014;5(2):91-8.

[30] Wong T, Ma A, Liu K, et al. Balance control, agility, eye-hand coordination, and sport performance of amateur badminton players: A cross-sectional study. Medicine. 2019;98(2):e14134.

[31] In T, Lee K, Song C. Virtual Reality Reflection Therapy Improves Balance and Gait in Patients with Chronic Stroke: Randomized Controlled Trials. Med Sci Monit. 2016;22:4046-53.

[32] Choi W, Han D, Kim J, Lee S. Whole-Body Vibration Combined with Treadmill Training Improves Walking Performance in Post-Stroke Patients: A Randomized Controlled Trial. Med Sci Monit. 2017;23:4918-25.

[33] Menz HB, Latt MD, Tiedemann A, et al. Reliability of the GAITRite walkway system for the quantification of temporo-spatial parameters of gait in young and older people. Gait Posture. 2004;20(1):20-5.

[34] Shin JW, Song GB, Ko J. The effects of neck and trunk stabilization exercises on cerebral palsy children's static and dynamic trunk balance: case series. J Phys Ther Sci. 2017;29(4):771-4.

[35] Keshner EA, Woollacott MH, Debu B. Neck, trunk and limb muscle responses during postural perturbations in humans. Exp Brain Res. 1988;71(3):455-66.

[36] Dubey L, Karthikbabu S, Mohan D. Effects of Pelvic Stability Training on Movement Control, Hip Muscles Strength, Walking Speed and Daily Activities after Stroke: A Randomized Controlled Trial. Ann Neurosci. 2018; 25(2):80-9.

[37] Tyson SF. Trunk kinematics in hemiplegic gait and the effect of walking aids. Clin Rehabil. 1999;13(4):295-300.

[38] Kong SW, Jeong YW, Kim JY. Correlation between balance and gait according to pelvic displacement in stroke patients. J Phys Ther Sci. 2015;27(7):2171-4.

[39] Park BS, Kim JH, Kim MY, et al. Effect of a muscle strengthening exercise program for pelvic control on gait function of stroke patients. J Phys Ther Sci. 2015;27(3): 641-4. 
